\title{
Formación profesional de enfermería durante la primera mitad del Siglo xx en la UNAM
}

\section{Nursing professional formation in the UNAM during the first half on the $20^{\text {Th }}$ century.}

Mtra. María Cristina Castañeda Godínez•

Mtra. Luz Pérez Loredo Díaz•

Mtra. Iñiga Pérez Cabrera

Mtra. María Cristina Müggenburg Rodríguez Vigil

\section{Preámbulo}

Además de los 200 años de la Independencia de México, los 100 años de la Revolución Mexicana, se conmemora el Centenario de la Universidad Nacional Autónoma de México, asociado a éste último es el centenario de la formación universitaria de los profesionales de Enfermería en México, 1911-2011. Esta celebración conmemorativa del "Centenario de la Enfermería Universitaria" es una ocasión para pensar, proceso que es medular en la enfermería contemporánea, la cual está inmersa en el mundo tecnificado, con dominio de la ciencia, lo que le dificulta el pensar incesante y vigoroso que impulse a salvaguardar la esencia, en este caso de los profesionales de esta disciplina ${ }^{1}$, manteniendo despierto ese pensar reflexivo al construir en el presente lo que serán las bases de la en- fermería que ahora soñamos, para que ese sueño sea una realidad en un futuro cercano.

\section{Antecedentes}

DE LA PROFESIONALIZACIÓN

DE LA ENFERMERÍA

Luz Pérez Loredo Díaz menciona ${ }^{2}$ que diversos acontecimientos posteriores a la conquista de México $\mathrm{y}$ anteriores al siglo $\mathrm{xx}$ han dejado testimonio del cuidado de los enfermos, considerado éste como la máxima aspiración de un grupo de personas dedicadas primordialmente a la asistencia hospitalaria, en todos aquellos establecimientos creados con este propósito a partir del siglo XVI, lo que permitió la profesionalización de la actividad del cuidado de enfermería. Esto es comprensible puesto que el dolor asoma ya desde los orígenes de la Humanidad, es lógico que hasta ellos alcance el instinto de preservación de la salud y el cuidado de las personas porque la enfermería como actividad del cuidado siempre ha acompañado al hombre. ${ }^{3}$ Esa labor que, por lo menos en los tres siglos anteriores, se ejerció en México, dejó antecedentes de que se aplicó con un sentido favorable hacia la población doliente, cuyos ejemplos se proyectaron no sólo para el momento en que se sucedieron sino que sembraron las raíces para dar los frutos en el futuro, futuro que hoy disfrutamos nosotros ${ }^{2}$

La tendencia predominante en la enfermería tuvo su base en el desarrollo de actividades acordes al momento histórico de la ciencia y la tecnología con grandes dotes de humanismo de quienes la ejercieron. Desde la alta edad media, con

\section{cैือง}

- Profesora ENEO-UNAM

-. Historiadora cronista de la FES Zaragoza.

... Profesor de carrera asociado C. de T. C ENEO-UnAM

... Maestría en Salud Pública y Profesor titular A DE T.C. ENEO-Unam

Correspondencia: inigapc@yahoo.es 
el acceso del cristianismo al poder del Estado, las profesiones se erigieron en torno a la fe cristiana, como es el caso de Enfermería. Con manuales de incipiente calidad y de escasa inclinación técnica, dieron una conformación a lo que más tarde sería considerado como un "perfil de enfermería."4

En el México independiente, la Escuela de Ciencias Médicas y posteriormente el Consejo General de Salubridad controló el ejercicio de las profesiones de la salud sin considerar a la enfermera, quien en su condición de mujer no era visible su actividad de cuidado. Aún en los inicios del Siglo xx, el Dr. Manuel Flores ${ }^{5}$ relata cómo los grupos conservadores consideraban a las mujeres incapaces de estudiar una profesión, porque su ingreso a las universidades era la ruina del hogar. La conveniencia imponía entre el hombre y la mujer la división del trabajo, la mujer a quien se le delegaban las funciones de la crianza, fue requerida en la asistencia en el hospital para el desempeño de funciones similares a las del hogar.

En 1860, fueron promulgadas las Leyes de Reforma, cuyo objetivo era controlar el poder que ejercía el clero, las que se ejecutaron a la caída de la invasión francesa y del imperio Francés. Una vez restaurada la República, el positivismo traído por el Dr. Gabino Barreda, en esos años sirvió como filosofía para organizar al país, también se aplicó al sistema educativo y después serviría para legitimar al Porfiriato. ${ }^{6}$

Durante este período de la Reforma, la función del cuidado enfermero con las Leyes de Desamortización de los Bienes de la Iglesia empezó a trasladarse a civiles, hasta que finalmente, en 1877, con la sa- lida de las hermanas de la Caridad, los hospitales pasaron a depender de la Beneficencia Pública y cobraron fuerza las ideas de salubridad y seguridad social. Sin embargo, los hospitales al perder las aportaciones de la Iglesia, no contaban con recursos humanos y materiales, lo cual puso en evidencia las carencias en la atención que se brindaba a los enfermos. Al acentuarse la crisis en la atención hospitalaria, por los movimientos políticos de las últimas décadas del siglo XIX, improvisaron personas en tareas de Enfermería, las cuales no tenían una preparación específica, y con frecuencia, tampoco poseían los atributos más esenciales para desempeñar un servicio que demandaba conocimientos científicos y técnicos que caracterizaban el avance de la medicina en los hospitales de la época ${ }^{2}$

Ya muy avanzado el siglo XIX, en el Hospital de Maternidad e Infancia y en el Hospital de San Andrés, daría inicio la propuesta de autoridades médicas de reconocido prestigio, un intento de mejoría en la preparación de aquellas personas que ejercieran la atención de enfermería a los pacientes hospitalizados. Fue en este momento

\begin{tabular}{|} 
Además de los 200 años \\
de la Independencia \\
de México, los 100 \\
años de la Revolución \\
Mexicana, se conmemora \\
el Centenario de la \\
Universidad Nacional \\
Autónoma de México \\
\hline
\end{tabular}

cuando en nuestro país ingresó la técnica de enfermería americana y europea, pues fueron contratadas enfermeras extranjeras para hacerse cargo de adiestrar a un grupo seleccionado de mujeres para este propósito. ${ }^{2}$

El proyecto de profesionalización requería de cambios en la estructura que se dieron desde 1900, cuando se inició la capacitación de las enfermeras que laboraban en el Hospital de San Andrés, en la Ciudad de México; con una formación programada y sistemática, impartida por enfermeras de formación superior. ${ }^{7}$ De 1900 a 1905, fueron varias las estrategias del grupo médico para reunir un grupo de estudiantes, que tuvieran, entre otras cualidades: un determinado grado de estudios. La modernización del país, consideró como la tarea central del gobierno, dadas las exigencias del mercado mundial y de las inversiones extranjeras a partir del Porfiriato, la adecuación de la Enfermería a esos requerimientos mediante la contratación de enfermeras alemanas y estadounidenses y la creación de programas educativos y modelos de profesionalización de la práctica de la Enfermería ${ }^{7}$.

\section{AnteCEDENTES DE LA FORMACIÓN EN LA ENFERMERÍA UNIVERSITARIA}

Dos años después de la inauguración del Hospital General de México en 1905 , se vio la necesidad de incrementar el número de personal ante las demandas de atención de los servicios, el 9 de febrero de 1907, se inauguró la Escuela de Enfermería en este mismo Hospital, considerando la necesidad de proveerlo de Enfermeras instruidas y convenientemente educadas, sien- 
do el Director de la misma el Dr. Fernando López.

Una de las principales deudas que tiene México para el Hospital General es la creación de enfermeras de carrera al planear la formación de enfermeras preparadas. En la plantilla de personal del Hospital General de México en 1905, las parteras ocupaban el nivel más alto, siguiendo la jefa de enfermeras, la enfermera segunda jefe, las enfermeras primeras, las enfermeras segundas. ${ }^{7}$

Para ese entonces el Dr. Liceaga había dicho:

"La asistencia de los enfermos va a confiarse a personas del sexo femenino, a quienes se ha estado dando desde hace dos años instrucción y educación apropiadas al objeto a que se les destina. La Escuela de Enfermeras puede considerarse, no sólo como un beneficio para los pobres que vienen a buscar su curación a este Hospital, sino como un poderoso factor de la difusión de conocimientos útiles a la mujer."

El Dr. Fernando López declaró:

"Así es que la verdadera Escuela de Enfermeras comienza ahora que hay Hospital que reúne todas las condiciones para la enseñanza. Esperamos confiadamente en que nuestro Gobierno sostendrá y mejorará la Escuela de Enfermeras, destinada no solamente a proveer de ellas al Hospital, sino permitir a las familias que utilicen los servicios de un personal inteligente y bien educado, en la asistencia domiciliaria, confiando a manos expertas los cuidados que ahora sólo prodiga el afecto, pero no siempre con la serenidad que reclama la delicada asistencia de los enfermos."
En 1908, Eulalia Ruiz Sandoval egresada de esta escuela de Enfermería del Hospital General es la primera mexicana, subjefe de enfermeras y en 1910 pasa a ser la primera jefe de enfermeras egresada de esta escuela. ${ }^{2}$ En cuanto al reglamento de la Escuela de Enfermería, éste estipulaba que el director del Hospital sería el director de la Escuela por lo tanto el doctor Fernando López fue director de la misma. La escuela de Enfermería dependió por corto tiempo de la Beneficencia Pública y, en 1908 pasó a la Secretaría de Instrucción Pública y Bellas Artes. La gestión de la escuela pudo permanecer por el desempeño de las enfermeras quienes demostraron que los servicios y el cuidado de los pacientes estaban garantizados; la formación de las enfermeras se diversificó y se vio la necesidad de que la escuela estuviera fuera del hospital, aunque la práctica desde el inicio de la profesionalización fue el eje de la formación. ${ }^{3}$

\section{La Universidad Nacional de MÉxico y La ENFERMería UNIVERSITARIA}

El 22 de septiembre de 1910, nació la Universidad Nacional de México impulsada por Justo Sierra, inaugurada por el Presidente de la República Don Porfirio Díaz y el Primer Rector Joaquín Eguía Lis. ${ }^{10}$ Se creó el Consejo Universitario al integrarse en una sola institución las Escuelas Nacionales de Medicina, Jurisprudencia, Bellas Artes, Altos Estudios y la Escuela Nacional Preparatoria. En ese momento, nadie podría prever que antes de dos meses diera inicio el movimiento armado de la Revolución Mexicana, que duraría 10 años y costaría un millón de muertes, con lo que México sería otro. ${ }^{10}$

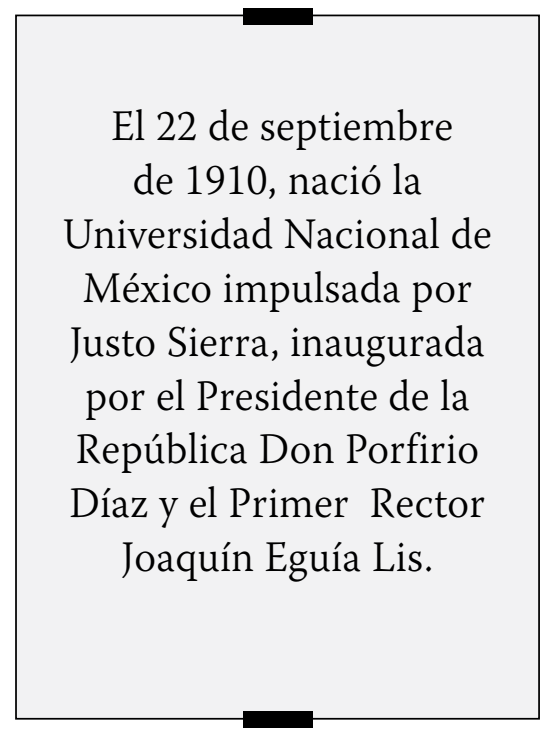

En 1911 se llevó a cabo la incorporación de la Escuela de Enfermería del Hospital General de México a la Escuela de Medicina de la Universidad. El ingreso de las enfermeras a la Universidad en 1911 fue la oportunidad única para salir del espacio unidimensional del Hospital-Escuela y liberarse de la uniformidad de los modelos sociales, para descubrir la polivalencia de las corrientes del pensamiento, su diversidad, su contradicción, su oposición, así como las diferentes opciones sociales, económicas y políticas que la sustentan. ${ }^{11} \mathrm{El} \mathrm{lu-}$ gar para la profesionalización de la enfermería en la universidad estaba definido, faltaría recorrer un largo tramo de más de 45 años para constituirse como disciplina científica e ingresar a la enseñanza superior, es decir,"...se inicia en el caminar interrogativo, que obliga al cuestionamiento y a la argumentación, permitiendo el dominio del pensamiento escrito." ${ }^{11}$

El 28 de junio de 1911, el Presidente Interino, Francisco León de la Barra, dispuso que la Escuela de Enfermería pasara a depender de la naciente Universidad Nacio- 
nal de México, y el 30 de diciembre de 1911 la Universidad de México aprueba integrar la enseñanza de la Enfermería a la Escuela de Medicina en las propias instalaciones de ésta. ${ }^{12}$ Durante esa época se instituyeron las carreras de enfermería y obstetricia con duración cada una de dos años, y en el caso de la obstetricia no tenía el antecedente de enfermería. $^{13}$

De 1911 a 1944 la Escuela de Enfermería y Obstetricia dependió de la Escuela de Medicina, estando ubicados los dos grupos en los hospitales sede: Hospital Juárez y el Hospital General de México. El doctor Fernando Zárraga director de la Escuela de Medicina, quien se desempeñó en este cargo del 6 de mayo de 1911 a 29 de abril de 1912, cambió la dependencia de la Escuela de Enfermería del Hospital General, a la Escuela de Medicina, con lo que se consolidó el nacimiento de ésta como escuela universitaria. ${ }^{7}$ Así la Escuela de Enfermería y Obstetricia compartió las mismas normas y reglamentos de la Escuela de Medicina derivados de la Universidad Nacional de México.(UNAM) El 11 de enero de 1912, se aprobó el Plan de Estudios para la carrera de Enfermera y la carrera de Partera de la Escuela de Enfermería y Obstetricia de esta naciente Universidad elaborado por los médicos.

En los hospitales sede, el Hospital General de México ya contaba con la estructura administrativa de control escolar por el antecedente de la Escuela de Enfermería, para la formación de recursos de enfermería y de obstetricia; pero en el Hospital Juárez las condiciones eran otra historia, desde la parte física con la escasez de recursos y la de- manda de servicios por la especialidad de urgencias y traumatología que lo caracterizaron desde el inicio de sus funciones en 1847 por la derivación de los pacientes de los movimientos bélicos como el de la Decena Trágica que vivió la capital de México. En 1911, al inicio de la Revolución Mexicana se creó la Cruz Blanca neutral, con la enfermera Ma. Elena Arizmendi Mejía en auxilio de los heridos en campo de batalla que después eran referidos al Hospital Juárez. Sin embargo, en este hospital, al mismo tiempo hubo decremento de pacientes de otro tipo de afecciones, por lo que a sugerencia del Director de la Beneficencia Pública, Licenciado Francisco C. García, ${ }^{14}$ se procedió al arreglo de una sala de obstetricia, que estuvo lista para comenzar a recibir parturientas el 30 de marzo de 1914 y las alumnas de enfermería pudieron continuar los estudios de Partera en ese hospital.

En el Periodo de 1911-1916 la inestabilidad política también se vio reflejada en el gobierno de la Universidad Nacional con los cambios frecuentes de rectores y por ende, de los directores de la Escuela de Medicina ${ }^{15}$. En esos periodos, se distinguieron por su actividad, el Dr. Aureliano Urrutia quién pretendió formar una Escuela esencialmente práctica con énfasis en la clínica, Don Ángel Hidalgo, hombre reflexivo, espíritu ponderado y disciplinado, de vasta cultura científica, pensó en la urgente necesidad de ampliar el edificio, con el fin de instalar aulas y laboratorios. Para ello compró la casa que perteneció a Don Ramón Fernández y que ocuparon más tarde la Escuela Odontológica y la de Economía, y ahora de la Facultad de Medicina.
Por etapas, realizó: la renovación del profesorado, la revisión de métodos y programas: la formación de un plan adecuado al tiempo y las circunstancias; el mejoramiento de los trabajos en las clínicas y laboratorios, etc. ${ }^{16}$

En 1916, el rector de la Universidad don José Natividad Macías, nombró director de la Escuela de Medicina al Dr. Rosendo Amor, distinguido ginecoobstetra; y más tarde fue nombrado secretario de la Escuela, el doctor Fernando Ocaranza, profesor de Fisiología. Durante su gestión se revisó el plan de estudios adaptándolo a las circunstancias del momento y estableció normas disciplinarias como fueron: rehusar inscripción a los que fuesen reprobados tres veces en la misma asignatura, que no fuesen examinados en cualquier materia, sin aprobación de las que formasen el año inmediato anterior; impulsado por su ideal de mejorar y transformar, fundó las cátedras de clínica terapéutica médica y clínica terapéutica quirúrgica y reinstaló la de Deontología con el nombre de moral médica $^{7}$. Por primera vez, en la vida de la Escuela, los trabajos prácticos fueron sometidos a un sistema preconcebido, para ser valorados y conceptuados como una condición indispensable de cumplimiento del plan de estudios, estipulando el número de trabajos perfectamente determinado, con el fin de adquirir derecho a examen. El principio era fundamental y terminante: nadie podría examinarse de materia práctica ni de materia teórico práctica, sin haber llevado a cabo el número de pruebas prácticas que la dirección fijaría al terminar cada año escolar? 
Don Guillermo Parra (15 de mayo de 1920 al 21 de junio de 1923), mejoró la enseñanza por medio de un aumento considerable de personal docente y la fundación de una policlínica anexa a la Facultad. Ya en este tiempo, se llevaba a cabo la selección de profesores por concurso de oposición. Gestionó obras materiales con el fin de ampliar la capacidad del edificio. ${ }^{16}$ El Dr. Don Manuel Gea González considerado como uno de los porta-estandartes del "pensamiento fisiológico", tomó a su cargo la dirección de la Escuela de Medicina el 21 de junio de 1923 al 1º de enero de 1925. Fundó la cátedra de fisiología patológica, a cargo del doctor José Palacios Macedo. El Dr. Don Fernando Ocaranza de 1925 a 1933, según afirma Fernández de Castillo, llegó "con el propósito inquebrantable de implantar el pensamiento rector de aquellos días, el pensamiento fisiológico y de orientar los estudios de la Facultad bajo esa égida". "Sabía muy bien que aquello sería motivo de luchas y provocación de enemistades: todo lo aceptamos a sabiendas y por ello mismo, ni ahora, ni entonces nos dolimos de que uno y otro hayan sucedido". ${ }^{15}$

Ya en el periodo posrevolucionario, en 1920, una vez constituido el México contemporáneo ${ }^{7}$, se dieron tres vertientes en el desarrollo de la profesión: la de la partera, la enfermera sanitaria y la enfermera hospitalaria y asistencial; visto desde el campo de trabajo. Como se mencionó con anterioridad, la preparación en el caso de la partera dependió de la Escuela de Medicina desde $1867^{7} y$ las enfermeras en instituciones reconocidas como la Universidad Nacional de México desde 1911 al incorporarse las

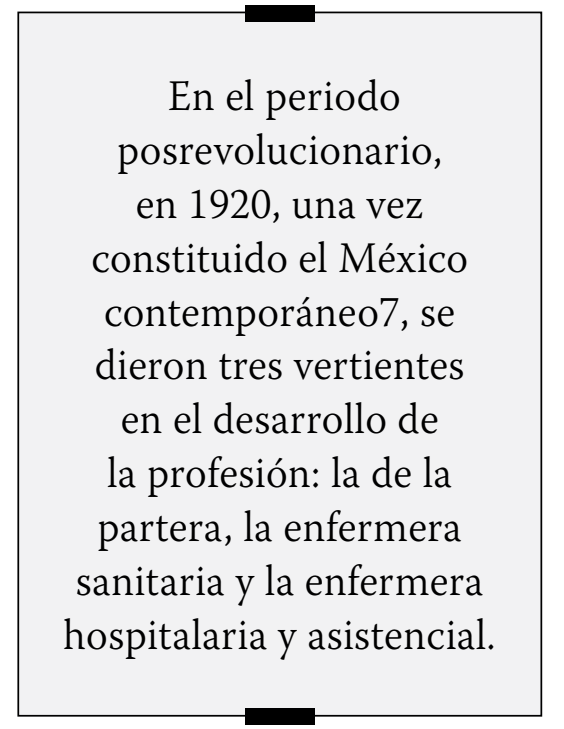

dos carreras de enfermera y partera con los mismos requisitos de ingreso, la enfermera sanitaria era formada por organismos del Sector Salud.?

En el ámbito laboral, se inició el reconocimiento al desempeño de las enfermeras, por su contribución a la consolidación de los sistemas de salud.

"El nacimiento del homenaje a las enfermeras se originó en el Hospital General de México, el 25 de diciembre de 1921, relata Soledad Amézquita, alumna de esas primeras generaciones de la escuela de Enfermería ${ }^{17}$ posteriormente el Dr. Castro Villagrana fijó el 6 de enero. El Dr. Luis de la Rosa fue el principal animador de este festejo, en años posteriores. En 1946, el homenaje a las enfermeras se llevó a efecto en la calle de Ayuntamiento, en la XEW, donde artistas, cantantes y el Dr. de la Rosa, amenizaban el acto. En 1965, por disposición presidencial se festejó el Día de la Enfermera a nivel nacional, en el Centro Médico Nacional, con la participación de la SSA, IMSS e ISSSTE, por disposición expresa del Presidente Díaz Ordaz."17
Dos años después de haberse implantado la enseñanza secundaria en el sistema educativo de México, en 1927, se dieron los primeros pasos para elevar la calidad de los estudios de las aspirantes a la carrera de enfermería, con el curso preparatorio de un año en el que se estudiaba: biología, matemáticas, química y física. Estas asignaturas orientaban la mejor comprensión de los conocimientos de la carrera después de la primaria en la Escuela Nacional Preparatoria. ${ }^{7}$

La década de los años veinte trajo a la Universidad el acontecimiento más importante para su futuro académico y social, el otorgamiento de su autonomía por el Presidente de la República Emilio Portes Gil, por lo cual desde 1929 se denomina Universidad Nacional Autónoma de México ${ }^{10}$, logro conseguido después de una larga lucha. Con la autonomía, la Universidad adquirió el status jurídico que le permitió regular sus propios asuntos académicos y administrativos sin injerencia del gobierno, el cual se comprometió a otorgar anualmente el presupuesto ${ }^{11}$. La Escuela de Medicina cambia la denominación a Facultad de Ciencias Médicas y en la Escuela de Enfermería se reestructura el plan de estudios de la carrera de Enfermería a tres años y la de Partera en dos años. ${ }^{7}$

En 1932, Carmen Gómez Siegler fue becada para ir a EEUU a estudiar la especialidad de enfermería quirúrgica y al regreso en 1933, se desempeñó como Jefe de enfermeras del Hospital General. Con apoyo del Dr. Luís Méndez reorganizó el departamento de enfermería, suprimió el internado, régimen que mantenía a las enfermeras las 24 horas del día en el hospital. 
Redujo la jornada a 12 horas, 4 más de las señaladas por una ley todavía incumplida y junto con Carmen Leija Paz de Ibarra organizó el festejo, en Navidad para Enfermeras. ${ }^{7}$

En el Hospital Juárez, con el comienzo del año 1933, fue implantado un servicio al que se le dio el nombre de "Hoja de Salud de la Enfermera", y que consistía en la realización periódica de exámenes a las enfermeras del Hospital, con el fin de mantenerlas en buen estado de salud y propiciar la atención de calidad a los pacientes. ${ }^{14}$ Ese mismo año, se realizó el Primer Congreso de Enfermeras y Parteras, organizado por Celia Alvarado de Reyes Campillo, Esperanza Pavía y Eloisa Muzquiz Pérez; de este Congreso de Enfermería surgió la idea de que se hiciera la secundaria previa a la carrera de Enfermería, así como para estudiar obstetricia cursar primero enfermería. ${ }^{7}$

En la asamblea Nacional de Cirujanos, 1934, las enfermeras y parteras de la UNAM discutieron sobre la necesidad de elevar el nivel social y preparación de las enfermeras. En 1935, el Dr. Ignacio Chávez señaló que el requisito para cursar enfermería sería tener la secundaria y haber cursado la carrera de Enfermería para realizar obstetricia. ${ }^{?}$

\section{El Dr. Ignacio Chávez EN LA FORMACIÓN PROFESIONAL DE ENFERMERÍA. $1935-1938$}

La trayectoria del Dr. Ignacio Chávez en la dirección de la Escuela Nacional de Medicina incluyó una acción dirigida a elevar el nivel de las alumnas de enfermería. Se exigió que las aspirantes tuvieran estudios de secundaria. El Dr. Ignacio Chávez se quejaba de que se culti- vara "el empirismo de la profesión" al no exigirse requisito alguno a las aspirantes a enfermeras. A partir de 1938 se exigió a las aspirantes, la presentación de su comprobante de estudios secundarios y la boleta de inscripción a los cursos profesionales en la Escuela de Medicina. Este requisito fundamental, tardó en implantarse, pues había una enorme necesidad de enfermeras y auxiliares, y las escuelas de enfermería no podían satisfacerla. Al instituir la secundaria como requisito de ingreso se planeaba romper el empirismo del que muchos médicos se quejaban. Díaz de Kuri y Viesca Treviño enfatizan: "Sin embargo, debe de confesarse, que ese empirismo profesional que se criticaba en 1938 produjo un grupo de enfermeras que puede catalogarse entre las mejores, sin importar el sitio, ni la latitud en el que se colocaran. Un equipo de primeras; es decir, de jefas de enfermeras de cada pabellón, hacía que todo marchara bien, con orden, haciendo que el trabajo se realizara eficaz y cumplidamente, vigilando a estudiantes y practicantes recién llegados e iniciándolos en las rutinas del hospital y, muchas veces, en la dinámica de algunos

En 1935, el Dr. Ignacio Chávez señaló que el requisito para cursar enfermería sería tener la secundaria y haber cursado la carrera de Enfermería para realizar obstetricia. tratamientos, administrando y estirando las exiguas dotaciones con que contaban."

El Hospital General no sería el mismo sin sus enfermeras y no queremos pasar adelante, sin mencionar los nombres de algunas de ellas: Brígida Bonilla, Carmen Pérez, Sofía Rosas, Ángela Cortés, Matilde Vera, María Elena Hernández, Guadalupe Maldonado, Isabel Pitman, María Luisa Saldivar, Rebeca Chumacera, Soledad Amézquita, Concepción Arteaga, Luz Maldonado, Celia Aguirre, Guadalupe Espinosa, Soledad Camarena, Guadalupe Lemus, Cipriana Flores, Esperanza Gómez, Marcelina Morales, Esperanza Martínez, Melita Velasco, Concepción Pérez, Otilia Márquez, Elena Tapia, Esperanza Velasco, Rita Ortiz, Josefina Labastida, Francisca Reyes; enérgicas y dulces a la vez, duras cuando era necesario, hábiles con las manos y con el entendimiento. ${ }^{9}$ Todas ellas egresadas de la Escuela de Enfermería y Obstetricia de la UNAM.

Honda huella dejaron las enfermeras en algunos de los médicos del hospital, "a ellas les debo mucho de lo que sé", refirieron en las entrevistas realizadas ex profeso para la preparación del libro Historia del Hospital General de México, personalidades tan diversas como lo son Jorge Flores Espinosa, Enrique Flores Espinosa, Salvador Medina Romo, Armando Ordóñez y Carlos Viesca Arrache. Muchas de ellas vivieron y murieron en su querido hospital, como fue el caso de Cipriana Flores, la primera del pabellón 20, quien ingresó a él en 1938 y murió internada allí mismo, 20 años después; o cómo Chelo Sánchez, una enfermera quirúrgica como ha habido pocas, de quien se 
puede decir que era toda una demostración de maestría como instrumentaba, sobre todo cuando lo hacia con cirujanos de la pericia, velocidad y exigencia del Dr. Clemente Robles; dicha enfermera murió a causa de un tumor cerebral, en el pabellón 32 del hospital, ya en los años setenta ${ }^{9}$

En 1936 se crearon los primeros cursos de especialización en Anestesiología y el de Instrumentación para enfermeras en el Hospital Juárez de México. ${ }^{18}$ Ese mismo año, otro evento importante fue la organización del Instituto de Convenciones Nacionales de Enfermeras en este mismo hospital.

En 1937 se desarrollaron en el Hospital General de México los cursos de especialización dirigidos a enfermeras tituladas, de anestesia y dietética, y las que obtuvieron las mejores calificaciones fueron ascendidas a la categoría de enfermeras especialistas. ${ }^{7} \mathrm{Al}$ año siguiente se continúa el Curso de instrumentistas con el Dr. Julián González Méndez y la Enfermera Instrumentista Lourdes Reyes Pavón. Las enfermeras egresadas fueron Leonor Molina, Rebeca Chumacero, Otilia Márquez González, Emma Montes de Oca y Guillermina Hernández y del curso de anestesia Piedad Sánchez Gavito, quien administró anestesia en circuito cerrado. En 1941 inició el curso de Enfermería Pediátrica del que fue egresada la Enfermera y Partera Reynalda Pineda Serino profesora de la ENEO en $1948 .^{9}$ Se hace notar que fueron egresadas de la escuela de Enfermería de la Universidad.

Las políticas de salud de 1936 a 1940, dieron mayor auge a la construcción de hospitales, y en la década de los '40 se crearon los de espe- cialidad, lo que trajo consigo mayor demanda de enfermeras y cambios en la atención a los pacientes, mejorando las condiciones laborales y económicas de las enfermeras. ${ }^{1}$ Ante este escenario, en 1944 se modificó el plan de estudios, incrementándose el número de asignaturas de 4 a 17 para la Carrera de Enfermera, materias que se iban perfilando a las nuevas necesidades de las especialidades, y a la carrera de Partera se le agregó la asignatura de Puericultura. ${ }^{7}$

En este nuevo Plan de Estudios, también se percibe un interés inicial por los aspectos psicológicos y la salud mental de las personas, sujetos de la atención de enfermería, así como la importancia de los aspectos éticos en el ejercicio de la profesión. Llama la atención que la asignatura de Trabajo Social se cursara en los tres años, situación que sugiere la relevancia que se le otorgaba a los aspectos sociales, como elemento explicativo del proceso salud enfermedad, igualmente del compromiso con la sociedad para contribuir a resolver sus problemas de salud.?

El final de este primer periodo de la escuela, corresponde, a la creación del IMSs y a una serie de hospitales de alta especialización: Institutos Nacionales de Cardiología, Nutrición, Cancerología, Neurología, Hospital Infantil y los de seguridad social: Hospital de PEMEX, Hospital de FERROCARRILES, mismos que determinaron un mayor énfasis en la práctica hospitalaria médica y de enfermería, demandando profesionales de enfermería con formación especializada, para las que se habían preparado recursos en los hospitales correspondientes. ${ }^{6}$

\section{Escuela Nacional de Enfermería y Obstetricia}

Los años de la década de los cuarenta, con el Presidente Manuel Ávila Camacho, fue más favorable al proyecto universitario y el Rector Mario de la Cueva pudo atender con dedicación una universidad más tranquila y solvente económicamente. En 1945 se publicó en el Diario Oficial una Nueva Ley Orgánica de la Universidad que suplió a la de 1929. En ella se especificaba, entre otros puntos, la autoridad de los directores de escuelas, facultades e institutos con los respectivos Consejos Técnicos para las primeras. ${ }^{10}$

De este proyecto nació la Escuela Nacional de Enfermería y Obstetricia (ENEO), con ese nombre está incluida en el Artículo $8^{\circ}$ del Estatuto General, y en el Artículo 45 que señala:"... cada Escuela y Facultad tendrá su propio Consejo Técnico, integrándose cada uno de ellos de acuerdo al Artículo 12 de la Ley Orgánica". Con este marco legal, la Escuela de Enfermería y Obstetricia se independizó de Medicina y a partir de entonces, se denominó Escuela Nacional de Enfermería y Obstetricia7. El primer Director fue el Dr. Everardo Landa, y celebró su primera reunión de Consejo Técnico con cinco profesores consejeros y dos consejeras alumnas. ${ }^{7}$ También en ese año el Congreso de la Unión aprobó el proyecto de ley para la Fundación y Construcción de Ciudad Universitaria. ${ }^{10}$

En la década de los cincuenta, se construyeron las principales obras de Ciudad Universitaria y se realizó una ceremonia de inauguración. En este proyecto universitario pese a las gestiones de la Dra. Emilia Leija Paz de Ortiz, Directora 


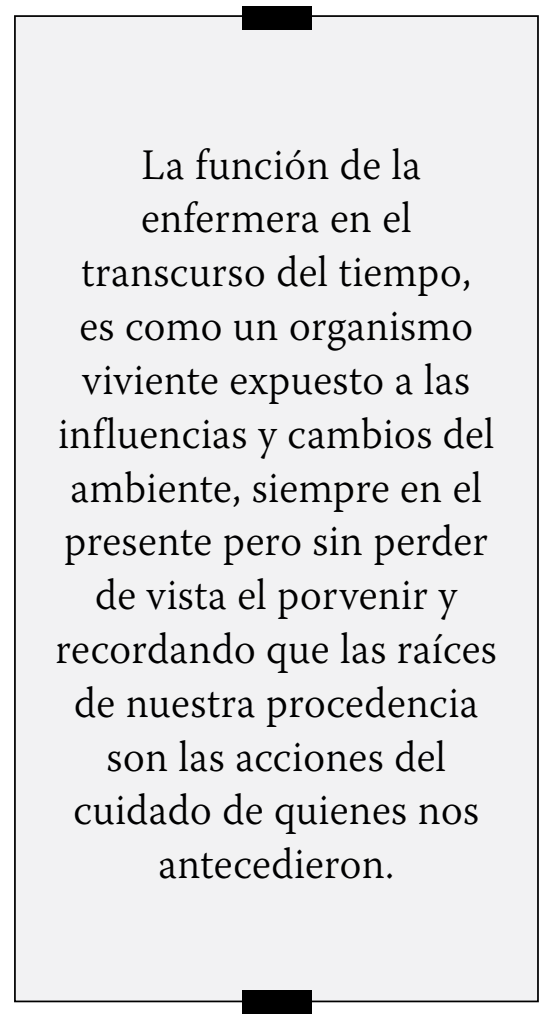

de la ENEO, no se incluyó a ésta dentro del campus de Ciudad Universitaria.

En 1952, la Escuela de Graduados organizó el Posgrado para Enfermeras Instructoras, auspiciado por la Universidad Nacional Autónoma de México y la Organización Mundial de la Salud, curso que logró incrementar la preparación de las instructoras y futuras profesoras de la disciplina de enfermería, dicho curso fue coordinado por la Enf. Hilda Lozier, de la Oficina Sanitaria Panamericana y la Enfermera Estela Corona, de la ENEO.

En 1953, la ENEO inició el nuevo proyecto para mejorar su programa educativo con el fin de ser incluida en el campus universitario; la OPS proporcionó material, equipo, y becas a ocho enfermeras instructoras para realizar estudios en el extranjero. El 6 de agosto de 1954, la Oficina Sanitaria Panamericana y la Organización Mundial de la Salud establecieron un convenio con México para realizar el proyecto de un nuevo plan de estudios de la Escuela Nacional de Enfermería y Obstetricia y satisfacer las peticiones hechas por las enfermeras mexicanas, a través de una encuesta nacional, previa al desarrollo del Seminario de Educación en Enfermería de Zimapán. El objetivo de este seminario fue "Estudiar la situación real de los servicios de enfermería del país, para que la preparación del personal se basara en las necesidades existentes y así conseguir el mejoramiento de los servicios de enfermería en toda la América Latina".

\section{ConcLusión}

La fuerza creada por el feminismo, concepto norteamericano que invitó a las mujeres a ocupar su lugar en todas las carreras y profesiones fue un parte-aguas en el desarrollo y empoderamiento de éstas en los distintos campos laborales y profesionales. La mitad del Siglo Xx dio a la enfermería un mayor prestigio que otras profesiones del área de la salud. La enfermera en la promoción de la salud, prevención y curación de los procesos morbosos ha ganado un lugar ante la sociedad que le da el reconocimiento desde lo político, lo laboral en los centros de trabajo de los distintos niveles de atención, del sector salud.

La función de la enfermera en el transcurso del tiempo, es como un organismo viviente expuesto a las influencias y cambios del ambiente, siempre en el presente pero sin perder de vista el porvenir y recordando que las raíces de nuestra procedencia son las acciones del cuidado de quienes nos antecedieron. Aunque la Enfermería Moder- na nace en la mitad del Siglo XIX con Florencia Nightingale, en donde la práctica de hospital fue importante, además de la sistematización de la educación. A partir de entonces y hasta el momento es de capital importancia la cultura y el espíritu que impregna las acciones de cuidado, dadas por el entendimiento y la sensibilidad lo que aunado al acervo de conocimientos ha tornado cada vez más en independiente y autónomo el ejercicio profesional de las enfermeras universitarias adecuado a la cambiante y compleja realidad dentro de la cual está enmarcada la institución social, la UNAM.

\section{Referencias bibLIOGRÁFICAS.}

1 Heidegger M. Serenidad. Gelanssenheit. Versión castellana de Yves Zimmermann. Barcelona: Ediciones del Serbal, 1994. p. 1

2 Pérez-Loredo DL. Características de los servicios de enfermería en el inicio del Siglo XX: Ens. Material bibliográfico para la materia de Historia de la Enfermería en México. México: ENEO-UNAM, 1986. p. 200.

3 De Mendijur LM. Enfermos, enfermeras y moral. Madrid: Ediciones Studium, 1964. p. 95.

4 Pérez-Loredo DL. La mujer en la Independencia y en la Revolución Mexicana. Conferencia. Encuentro de Enfermería del Estado de México. 20 de mayo de 2010. p. 1.

5 Flores M. La Mujer y las profesiones liberales. En: Bazant Mílada [comp.] Debate pedagógico durante el porfiriato, Antología. México: SEP/El Caballito, 1985. p. 143.

6 Pérez I, Müggenburg C, Castañeda C. Visión global del desarrollo de la práctica del cuidado de enfermería. En: La teoría de Enfer- 
mería. Dimensiones, conceptos y aplicaciones prácticas. [Libro Multimedia]. México: ENEO-UNAM, 2010. p. 15.

7 Pérez I. y Castañeda C. 100 años ENEO. 1907-2006. [CD interactivo]. México: ENEO-UNAM, 2006. Cap. 3, p 5

8 Fernández del Castillo F. El hospital General de México. Antecedentes y evolución. En: Pérez I y Castañeda C. [editoras]. 100 años ENEO. 1907-2006. [CD interactivo]. México: ENEO-UNAM, 2006. Cap. 4.p.5

9 Díaz de Kuri M. y Viesca Treviño C. Historia del Hospital General de México. En: Pérez I y Castañeda C. 100 años ENEO. 1907-2006. [CD interactivo]. México: ENEOUNAM,2006, Cap.4: p. 7

10 García HA. y Jiménez A. El sueño de Justo Sierra, Una Universidad para la Nación. En: Los 100 años de la UNAM. México: La Jornada, 22 septiembre 2010. p. 20.

11 Hernández CJ. Historia de la Enfermería. Un análisis histórico de los cuidados de enfermería. Madrid: McGraw-Hill - Interamericana de España. 1995. p. 142.
12 Pérez-Loredo DL. Efemérides de Enfermería 1900-1985. En: Pérez I y Castañeda C. [editoras]. 100 años ENEO. 1907-2006. [CD interactivo]. México: ENEO-UNAM, 2006. Cap.4. p. 8.

13 Guevara RA. Organización Académica 1974. En: Pérez I y Castañeda C. [editoras]. 100 años ENEO. 1907-2006. [CD interactivo]. México: ENEO-UNAM, 2006. Cap.4. p. 10.

14. Velasco R. El Hospital Juárez. Antes Hospital Municipal de San Pablo. En: Pérez I. Müggenburg C, Castañeda C. Visión global del desarrollo de la práctica del cuidado de enfermería. En: La teoría de Enfermería. Dimensiones, conceptos y aplicaciones prácticas. [Libro Multimedia]. México: ENEO-UNAM, 2010. Cap. 1: 21.

15 Lista de Directores Facultad de Medicina. Disponible en: http://www. facmed.unam.mx/fm/historia/lista.html Consultado en Octubre de 2005.

16 Ocaranza F. Breve Historia de la facultad de medicina de México. Pérez I y Castañeda C. [editoras]. 100 años ENEO. 1907-2006. [CD inte- ractivo]. México: ENEO-UNAM, 2006. Cap. 4, p 10.

17 Pérez- Loredo DL. Archivos personales. 2008. En: La teoría de Enfermería. Dimensiones, conceptos y aplicaciones prácticas. [Libro Multimedia]. México: ENEO-UNAM, 2010. Cap. 1: p. 24.

18 Morales Herrera MJ. Cronología de la Escuela Nacional de Enfermería y Obstetricia. UNAM. 1929-1979. En: Pérez I y Castañeda C. [editoras]. 100 años ENEO. 1907-2006. [CD interactivo]. México: ENEOUNAM, 2006. Cap.4. p. Ibid. Cap. 4 p. 23.

19 Ávila JR. La importancia del papel de enfermería de los programas de atención de la salud de México. Reseña histórica de la creación y evolución de la ENEO, 1911-1986. 75 Aniversario de la Escuela Nacional de Enfermería y Obstetricia de la UNAM, México. Compilación, revisión y publicación: Lic. Esther Hernández Torres. Portada Maestro Tomas Elguera, 1986 p. 79-81. 